\title{
Caracterización de los trastornos manométricos esofágicos en pacientes con enfermedad por reflujo gastroesofágico refractario con síntomas esofágicos
}

\author{
Characterization of esophageal motility disorders in refractory \\ gastroesophageal reflux disease patients with esophageal symptoms
}

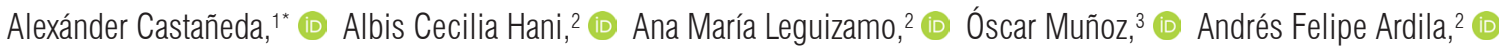
Valeria Atenea Costa. ${ }^{2}$

\begin{abstract}
GaCCESO ABIERTO
Citación:

Castañeda A, Hani AC, Leguizamo AM, Muñoz 0 , Ardila AF, Costa VA. Caracterización de los trastornos manométricos esofágicos en pacientes con enfermedad por reflujo gastroesofágico refractario con síntomas esofágicos. Rev Colomb Gastroenterol. 2021;36(2):212-217. https://doi. org/10.22516/25007440.678

Fellow de Gastroenterología y Endoscopia Digestiva, Pontificia Universidad Javeriana. Hospital Universitario San Ignacio, especialista en Medicina Interna. Bogotá, Colombia. Especialista en Gastroenterología y Endoscopia Digestiva, profesor, Pontificia Universidad Javeriana, Hospital Universitario San Ignacio, especialista en Medicina Interna. Bogotá Colombia.

3 Doctor en Epidemiología Clínica, especialista en Medicina Interna, Pontificia Universidad Javeriana, Hospital Universitario San Ignacio. Bogotá, Colombia.

*Correspondencia: Alexánder Castañeda-Ladino.
\end{abstract} alexander.castaneda@javeriana.edu.co

Fecha recibido: $\quad 16 / 10 / 20$ Fecha aceptado: 17/02/21

\begin{abstract}
Resumen
Introducción: la enfermedad por reflujo gastroesofágico (ERGE) refractaria puede conducir a complicaciones potenciales como la esofagitis persistente, estenosis esofágica, anillo de Schatzki y esófago de Barrett. Este estudio describe la motilidad en pacientes con ERGE refractaria y su relación con síntomas esofágicos. Método: se realizó un estudio observacional analítico a partir de una cohorte retrospectiva en pacientes con diagnóstico de ERGE refractaria y síntomas esofágicos a quienes se les realizó manometría esofágica de alta resolución más impedanciometría. Se describen las características clínicas y demográficas, y la asociación entre los trastornos manométricos y los síntomas esofágicos. Resultados: se incluyeron 133 pacientes (edad promedio: $54,1 \pm 12,5$ años). La pirosis y regurgitación $(69,2 \%)$ y la disfagia esofágica $(13,5 \%)$ fueron los síntomas más comunes. La motilidad normal $(75,2 \%)$, el aclaramiento completo del bolo $(75,2 \%)$ y la motilidad esofágica inefectiva (MEI) (18\%) fueron los hallazgos manométricos más frecuentes. La unión gastroesofágica tipos II y IIIb estuvieron presentes en el 35,3\% y 33,8 \% de los casos, respectivamente. La aperistalsis $(3,8 \%)$ y el esófago en martillo neumático (Jackhammer, $0,8 \%$ ) fueron infrecuentes. El aclaramiento incompleto del bolo se asoció con disfagia esofágica $(p=0,038)$ y a $\mathrm{MEI}(p=0,008)$. Ningún síntoma esofágico se relacionó significativamente con trastornos de motilidad. Conclusiones: los resultados de nuestro estudio sugieren que los trastornos de motilidad son infrecuentes en los pacientes con ERGE refractaria. Adicionalmente, sugieren que la presencia de alteraciones de motilidad esofágica no se relaciona con la presencia de síntomas esofágicos y, por tanto, que el tipo de síntoma presentado no permite predecir la existencia de dichos trastornos.
\end{abstract}

\section{Palabras clave}

Reflujo gastroesofágico refractario, manometría de alta resolución, motilidad esofágica.

\begin{abstract}
Introduction: Refractory gastroesophageal reflux disease (GERD) can lead to potential complications such as persistent esophagitis, esophageal stricture, Schatzki ring, and Barrett's esophagus. This study describes motility in patients with refractory GERD, and its association with esophageal symptoms. Materials and methods: An analytical observational study was carried out in a retrospective cohort of patients diagnosed with refractory GERD and esophageal symptoms who underwent high-resolution esophageal manometry and impedance testing. Clinical characteristics, demographics, and the association between motility disorders and esophageal symptoms are described. Results: 133 patients were included (mean age $54.1 \pm 12.5$ years). Heartburn and regurgitation $(69.2 \%)$, and esophageal dysphagia (13.5\%) were the most common symptoms. Normal motility $(75.2 \%)$, complete bolus clearance $(75.2 \%)$, and ineffective esophageal motility (IEM) $(18 \%)$ were the most frequent manometric findings. Type II and IIlb gastroesophageal junction were observed in $35.3 \%$ and $33.8 \%$ of the cases, respectively. Esophageal aperistalsis (3.8\%) and Jackhammer esophagus $(0.8 \%)$ were rare findings. Incomplete bolus clearance was associated with esophageal dysphagia $(p=0.038)$ and IEM $(p=0.008)$. No esophageal symptoms were significantly related to motility disorders. Conclusions: The results of the present study suggest that motility disorders are rare in patients with refractory GERD. They also suggest that esophageal motility disorders are not associated with the presence of esophageal symptoms and, therefore, the type of symptom experienced does not allow predicting the existence of such disorders.
\end{abstract}

\section{Keywords}

Refractory gastroesophageal reflux; High-resolution manometry; Esophageal motility. 


\section{INTRODUCCIÓN}

La enfermedad por reflujo gastroesofágico (ERGE) es una de las principales causas de atención médica en todo el mundo. Su prevalencia ha aumentado notablemente en las últimas décadas y es mayor en Norteamérica (19,8\%), en comparación con Asia Oriental (5,2\%), Medio Oriente $(14,4 \%)$, Europa $(15,2 \%)$ y Colombia $(11,8 \%)(1-4)$. Entre el $10 \%$ y el $40 \%$ de los pacientes con ERGE presentan reflujo refractario, definido como la persistencia de síntomas a pesar de la terapia óptima con inhibidores de la bomba de protones (IBP), a doble dosis, 30 a 60 minutos antes de las comidas durante al menos 8 semanas (5, 6). Esta refractariedad puede conducir a complicaciones potenciales como la esofagitis persistente, estenosis esofágica, anillo de Schatzki y esófago de Barrett que podría terminar en adenocarcinoma (1).

Estudios previos demuestran que se requiere el compromiso de 1 o más sistemas de protección para producir reflujo refractario y los principales son la integridad reducida de la barrera antirreflujo, ya sea mediante un esfínter esofágico inferior (EEI) hipotónico o un desplazamiento axial entre el esfínter, y el diafragma crural (hernia hiatal); el aclaramiento incompleto del contenido ácido y del bolo, el cual está alterado en pacientes con peristaltismo anormal y salivación deteriorada; la integridad reducida de la mucosa, con espacios intracelulares dilatados; $y$ el vaciamiento gástrico lento, que puede conducir a un aumento de la distensión gástrica y el inicio de eventos de reflujo a través de relajaciones transitorias del EEI. También se describen causas inherentes al procesamiento del fármaco, dependiente del polimorfismo genético de la actividad de CYP2C19; la presencia de bolsillo ácido; hipersensibilidad al reflujo; esofagitis eosinofílica, infecciosa o por píldoras; presencia de comorbilidades que condicionen el aumento de la presión intraabdominal, como la ascitis; y el uso de medicamentos que retrasen el tránsito gastrointestinal (5, 7-11).

En cuanto a los trastornos motores esofágicos (12), se ha descrito que una disminución de la capacidad del esófago para limpiar el contenido del reflujo termina por generar refractariedad al manejo (9). Los estudios publicados con respecto a la presencia de trastornos de motilidad en pacientes con reflujo refractario son limitados y no asocian estos hallazgos con síntomas esofágicos. Adicionalmente, no existen estudios en esta aérea de interés realizados en la población colombiana.

Este estudio describe los trastornos de motilidad y su relación con síntomas esofágicos (analizados por manometría esofágica de alta resolución) en un grupo de pacientes con reflujo refractario manejados en un hospital de referencia en Colombia.

\section{MÉTODOS}

Realizamos un estudio observacional analítico a partir de una cohorte retrospectiva. Se incluyeron todos los pacientes mayores de 18 años con reflujo refractario $(5,6)$ y síntomas esofágicos a quienes se les realizó manometría esofágica de alta resolución más impedanciometría entre el 1 de julio y el 31 de diciembre de 2019. Las pacientes en estado de gestación fueron excluidas. El proyecto fue evaluado y aprobado por el comité de ética del Hospital Universitario San Ignacio (HUSI) y la Pontificia Universidad Javeriana.

Los pacientes se identificaron a partir de la base de procedimientos del departamento de Gastroenterología y Endoscopia digestiva del HUSI, en Bogotá, Colombia. La información demográfica y los síntomas esofágicos se extrapolaron de un formato diseñado para tal fin, en el que se registran de forma sistemática los datos obtenidos en una encuesta realizada a todos los pacientes antes de la realización de la manometría esofágica. Dicho procedimiento se realizó con un equipo de Medtronic, Given Imaging (Medtronic, Los Ángeles, California, Estados Unidos).

De acuerdo con múltiples publicaciones que incluyen el consenso de Montreal, el diagnóstico de enfermedad por reflujo gastroesofágico se basó en parámetros clínicos (4, 13-15); es decir, síntomas típicos, o atípicos esofágicos como disfagia o dolor torácico en pacientes en quienes ya se descartó etiología coronaria (4). No incluimos síntomas extraesofágicos atípicos, decisión basada en un menor nivel de acuerdo, también expuesto en el consenso (4). Para definir los síntomas de refractariedad, se tuvieron en cuenta los mecanismos fisiopatológicos de refractariedad (incremento de las relajaciones transitorias del EEI, hernia hiatal, hipotensión del EEI, alteración de la contractilidad esofágica, incremento de la permeabilidad de la mucosa y retraso del vaciamiento gástrico), los cuales están relacionados con los síntomas de disfagia, dolor torácico no cardiogénico, pirosis y regurgitación (5). Los patrones de presentación de motilidad y los trastornos de motilidad esofágica se definieron de acuerdo con los criterios de Chicago 3.0 (12). Los síntomas esofágicos fueron definidos según las guías de diagnóstico y manejo de ERGE del American College of Gastroenterology (13). El sindrome de reflujo típico se definió como la presencia de pirosis y regurgitación.

Se describieron las características clínicas, demográficas y funcionales utilizando medidas de tendencia central y medidas de dispersión, de acuerdo con la distribución de los datos. Se usó una prueba de Shapiro-Wilk para evaluar el supuesto de normalidad. Las variables categóricas se reportaron como números absolutos y proporciones. Para evaluar la asociación entre la presencia de síntomas y los trastornos de motilidad, se usó una prueba de Chi-cuadrado 
$\left(\chi^{2}\right)$. Para el análisis estadístico se utilizó el paquete estadístico STATA 15.

\section{RESULTADOS}

Se incluyeron 133 pacientes, cuyas características demográficas y tipo de síntomas se muestran en la Tabla 1. La mayoría de los pacientes eran mujeres $(72,2 \%)$ con una media de edad de 54 años $( \pm 12,5)$. Todos los pacientes estaban en tratamiento con un IBP. El síntoma más frecuente fue pirosis y regurgitación $(69,2 \%)$, seguido de disfagia esofágica $(13,5 \%)$, disfagia orofaríngea $(12 \%)$ y, finalmente, dolor torácico no cardiogénico $(8,3 \%)$.

Tabla 1. Características demográficas de los pacientes y síntomas esofágicos

\begin{tabular}{|lc|}
\hline \multicolumn{1}{c}{ Variable } & $\mathbf{n}=133$ \\
\hline Edad, media (DE) & $54,1(12,5)$ \\
\hline Mujer, n (\%) & $96(72,2)$ \\
\hline Síntomas n (\%) & \\
\hline - Disfagia orofaríngea & $16(12,0)$ \\
\hline - Disfagia esofágica & $18(13,5)$ \\
\hline - Dolor no cardiogénico & $11(8,3)$ \\
\hline - Pirosis y regurgitación (síndrome de reflujo típico) & $92(69,2)$
\end{tabular}

DE: desviación estándar.

En la Tabla 2 se presentan los hallazgos y trastornos de motilidad. La motilidad normal y el aclaramiento completo del bolo fueron los más frecuentes (75,2 \% para cada uno). La MEI fue el trastorno más frecuente (18\%). Fue llamativa la presencia de aperistalsis en $3,8 \%$ y esófago en martillo neumático (Jackhammer) en $0,8 \%$ de los pacientes. Con respecto a los hallazgos manométricos, la unión gastroesofágica (UGE) tipos II y IIIb fueron las más frecuentes (35,3\% y $33,8 \%$, respectivamente), la hipotonía del esfínter esofágico inferior (EEI) se evidenció en un $15 \%$. Los demás trastornos de motilidad se presentaron en menos del 1,5\% de los pacientes.

La asociación entre los trastornos manométricos con pirosis y regurgitación se muestran en la Tabla 3. Al comparar los pacientes con estos síntomas, ninguno se relacionó significativamente con algún hallazgo o trastorno de motilidad y la mayoría tenía manometrías normales. Para los demás síntomas esofágicos, cabe resaltar que no se encontraron asociaciones significativas con algún tipo de hallazgo o trastorno de motilidad.
Tabla 2. Hallazgos en la manometría esofágica de alta resolución más impedanciometría

\begin{tabular}{lc}
\multicolumn{1}{c}{ Variables manométricas } & $\begin{array}{c}\text { Número de pacientes } \\
(\mathbf{n}=133)\end{array}$ \\
\hline Tipo de UGE, $\mathrm{n}(\%)$ & $25(18,8)$ \\
- I & $47(35,3)$ \\
- II & $16(12)$ \\
- IIla & $45(33,8)$ \\
- IIlb & $100(75,2)$ \\
Aclaramiento del bolo, $\mathrm{n}(\%)$ & \\
Acalasia, $\mathrm{n}(\%)$ & $0(0)$ \\
- I & $2(1,5)$ \\
- II & $0(0)$ \\
- III & $1(0,8)$ \\
Obstrucción al tracto de salida, $\mathrm{n}(\%)$ & $0(0)$ \\
Espasmo esofágico distal, $\mathrm{n}(\%)$ & $1(0,8)$ \\
\hline Esófago en martillo neumático, $\mathrm{n}(\%)$ & $5(3,8)$ \\
\hline Aperistalsis, $\mathrm{n}(\%)$ & $24(18)$ \\
\hline MEl, $\mathrm{n}(\%)$ & $0(0)$ \\
\hline Peristalsis fragmentada, $\mathrm{n}(\%)$ & $100(75,2)$ \\
\hline Motilidad normal, $\mathrm{n}(\%)$ & $20(15)$ \\
\hline Hipotonía del EEl, $\mathrm{n}(\%)$ &
\end{tabular}

EEI: esfinter esofágico inferior MEI: motilidad esofágica inefectiva; UGE: unión gastroesofágica.

Los pacientes con aclaramiento incompleto presentaron más disfagia esofágica que aquellos con aclaramiento completo $(24 \%$ frente a $10 \%, p=0,038)$. De igual forma, la MEI se asoció con un retraso del aclaramiento (33\% frente a $13 \%, p=0,008)$. Todos los pacientes con acalasia tipo II y aperistalsis tuvieron un retraso del aclaramiento del bolo. Entre los pacientes con motilidad normal, el $86 \%$ tuvo aclaramiento completo comparado con solo el $14 \%$ entre aquellos con alteración de la motilidad $(p<0,005)$.

\section{DISCUSIÓN}

La ERGE refractaria tiene un impacto en el riesgo de complicaciones, como esofagitis persistente, estenosis esofágica, anillo de Schatzki y esófago de Barrett que podría terminar en adenocarcinoma (1). Dentro de los factores condicionantes de refractariedad se encuentran las alteraciones de la barrera antirreflujo, deterioro del aclaramiento esofágico, integridad reducida de la mucosa, vaciamiento gástrico lento, causas inherentes a la farmacodinamia y trastornos de motilidad esofágica $(5,9)$. En el presente estu- 
Tabla 3. Asociación entre síndrome de reflujo típico con los trastornos manométricos

\begin{tabular}{|c|c|c|c|}
\hline $\begin{array}{l}\text { Variables esofágicas } \\
\text { manométricas }\end{array}$ & $\begin{array}{l}\text { Pirosis y } \\
\text { regurgitación } \\
\text { presentes } \\
(\mathrm{n}=92)\end{array}$ & $\begin{array}{l}\text { Pirosis y } \\
\text { regurgitación } \\
\text { ausentes } \\
(n=41)\end{array}$ & $\begin{array}{c}\text { Valor } \\
p\end{array}$ \\
\hline $\begin{array}{l}\text { Tipo de UGE, n (\%) } \\
-\quad \text { I } \\
- \text { II } \\
- \text { IIla } \\
-\quad \text { IIlb }\end{array}$ & $\begin{array}{c}16(17,4) \\
35(38) \\
10(10,9) \\
31(33,4)\end{array}$ & $\begin{array}{c}9(22) \\
12(29,3) \\
6(14,6) \\
14(34,5)\end{array}$ & 0,73 \\
\hline Aclaramiento del bolo, $\mathrm{n}(\%)$ & $71(77,2)$ & $29(70,1)$ & 0,42 \\
\hline $\begin{array}{l}\text { Acalasia, n (\%) } \\
-\quad \text { I } \\
-\quad \text { II } \\
-\quad \text { III }\end{array}$ & $\begin{array}{c}0(0) \\
2(100) \\
0(0)\end{array}$ & $\begin{array}{l}0(0) \\
0(0) \\
0(0)\end{array}$ & 0,34 \\
\hline $\begin{array}{l}\text { Obstrucción al tracto de } \\
\text { salida, } n(\%)\end{array}$ & $1(100)$ & $0(0)$ & 0,50 \\
\hline Espasmo esofágico distal, n (\%) & $0(0)$ & $0(0)$ & \\
\hline $\begin{array}{l}\text { Esófago en martillo } \\
\text { neumático, n (\%) }\end{array}$ & $1(100)$ & $0(0)$ & 0,50 \\
\hline Aperistalsis, $\mathrm{n}(\%)$ & $2(40)$ & $3(60)$ & 0,15 \\
\hline MEI, n (\%) & $19(20,6)$ & $5(12,2)$ & 0,24 \\
\hline Peristalsis fragmentada, $\mathrm{n}(\%)$ & $0(0)$ & $0(0)$ & \\
\hline Motilidad normal, n (\%) & $68(68)$ & $32(32)$ & 0,61 \\
\hline Hipotonía EEI, n (\%) & $13(14,1)$ & $7(17)$ & 0,66 \\
\hline
\end{tabular}

EEI: esfinter esofágico inferior MEI: motilidad esofágica inefectiva; UGE: unión gastroesofágica.

dio se encontró que la mayoría de los pacientes con ERGE refractaria no tiene trastornos manométricos asociados y entre los que presentan alguna alteración, la MEI fue la más frecuente. Adicionalmente, se encontró que la presencia de alteraciones de motilidad esofágica no se relaciona con la presencia de síntomas esofágicos.

La mayoría de nuestros pacientes fueron mujeres (72,2\%) con una edad media de 54,1 años, hallazgos concordantes con los reportados por Abdallah, quien encontró una mayor prevalencia de sexo femenino $(68,8 \%)$ y una edad media de 46,6 años (10). Por una parte, la manometría esofágica normal fue el hallazgo más frecuente $(75,2 \%)$ y la MEI, el trastorno de motilidad más común, lo que concuerda con lo descrito en publicaciones previas $(7,9,16)$. Por otra parte, nosotros encontramos que la UGE tipo II $(35,3 \%)$ y IIIb $(33,8 \%)$ fue el hallazgo más frecuente en relación con la presencia de hernia hiatal, resultados en proporción mayores a los reportados en otros estudios que documentan hasta $18 \%$ de hernia hiatal en el contexto de reflujo refractario (10), hallazgos que podrían estar en relación con el volumen de pacientes evaluados en nuestro estudio ya que el HUSI es un centro de referencia.

Llamó la atención la presencia de trastornos de motilidad mayor como el esófago en martillo neumático y la aperistalsis, aunque fueron infrecuentes (proporción menor de $3,8 \%)$. Publicaciones previas ya han encontrado asociación entre síntomas sugestivos de reflujo y trastornos de motilidad mayor, hallazgos que podrían ser explicados por insuficiencia en la peristalsis primaria o por una respuesta vigorosa y sostenida (espástica) ante la exposición anormal al reflujo $(6,8,9,16)$. Incluso, se ha planteado una probable secuencia evolutiva, desde un trastorno menor hasta uno mayor en el contexto de hipomotilidad (17). La motilidad espástica puede ocurrir concomitantemente o como consecuencia de otras afecciones, como la ERGE, lo que produce hipercontractilidad. Además, se ha descrito que pueden mejorar con la terapia de IBP $(18,19)$. Se puede considerar que el reflujo existe como una manifestación de un trastorno mayor de motilidad de base; sin embargo, como sabemos, el abordaje inicial se realiza como ERGE debido a su mayor prevalencia; por esta razón es importante identificar causas subyacentes de resistencia a los IBP.

Por una parte, la pirosis y regurgitación (síndrome de reflujo típico; 69,1\%) fueron los síntomas más frecuentemente asociados con reflujo refractario, hallazgos concordantes con los datos publicados por otros autores (10). Por otra parte, nuestros hallazgos sugieren que el tipo de síntoma no permite predecir si existe o no un trastorno de motilidad, lo que adquiere un significado clínico importante a la hora de evaluar estos pacientes. Patcharatrakul, al igual que Ala y colaboradores concuerdan con nuestros hallazgos en cuanto a que existe una pobre relación de síntomas típicos con algún tipo de dismotilidad esofágica, lo que sugiere que generalmente no hay contracción esofágica durante la aparición de estos síntomas $(16,17)$.

Es conocido que las alteraciones de la motilidad esofágica son factores relacionados con el aclaramiento del bolo. En concordancia con nuestro estudio, Roman y Bulsiewicz (20-22) demostraron que la separación espacial o ausencia de la peristalsis se asocian con un tránsito del bolo incompleto y probablemente con disfagia esofágica. Sin embargo, el aclaramiento anormal del bolo puede observarse en pacientes con motilidad normal, hallazgos también descritos por Bogte $(23,24)$. Por una parte, en cuanto a los trastornos de motilidad asociados con una presión de relajación integrada (IRP) elevada, estudios previos muestran que los pacientes con acalasia presentan alteración del aclaramiento del bolo, en comparación con los valores normales para los pacientes con obstrucción 
del tracto de salida, hallazgos semejantes a los descritos en nuestro trabajo. Esta herramienta secundaria podría ser de utilidad para diferenciar entre estas dos alteraciones (25). Por otra parte, se ha descrito que las contracciones fallidas y la contractilidad ineficaz se asocian con una eliminación incompleta del bolo y se ha demostrado que $30 \%$ o más contracciones fallidas y $70 \%$ o más contracciones ineficaces tienen la mejor sensibilidad y especificidad para predecir una eliminación alterada $(26,27)$.

Dentro de las fortalezas de nuestro estudio se destaca la cantidad de pacientes con diagnóstico de ERGE refractaria a quienes se les realizó manometría esofágica y la caracterización de trastornos de motilidad. Sin embargo, deben reconocerse limitaciones relacionadas con la naturaleza retrospectiva del estudio y la presencia limitada de algunos trastornos de motilidad, lo que limita la evaluación de la asociación con los síntomas evaluados. Además, utilizar parámetros clínicos para definir la presencia de reflujo gastroesofágico en ausencia de $\mathrm{pH}$ impedanciometría y endoscopia para la totalidad de los pacientes evaluados puede ser considerado como una limitación; sin embargo, de acuerdo con el consenso de Lyon (14), estos exámenes tienen una sensibilidad y especificidad de $70 \%$ y $67 \%$, respectivamente, lo que evidencia la similitud válida del rendimiento diagnóstico al compararlo con los criterios clínicos. Adicionalmente, nuestros resultados no podrán extrapolarse a pacientes que no vengan en tratamiento farmacológico. Se requerirán nuevos estudios multicéntricos para evaluar la asociación entre ERGE refractaria y los trastornos de motilidad con los hallazgos de la pH impedanciometría.

\section{REFERENCIAS}

1. Rubenstein JH, Chen JW. Epidemiology of gastroesophageal reflux disease. Gastroenterol Clin North Am. 2014;43(1):1-14. https://doi.org/10.1016/j.gtc.2013.11.006

2. Páramo DB, Albis R, Galiano MT, Mendoza de Molano B, Rincón R, Pineda Ovalle LF, et al. Prevalencia de síntomas del reflujo gastroesofágico y factores asociados: una encuesta poblacional en las principales ciudades de Colombia. Revista Colombiana de Gastroenterología. 2016;31(4):337-46. https://doi.org/10.22516/25007440.108

3. Eusebi LH, Ratnakumaran R, Yuan Y, Solaymani-Dodaran M, Bazzoli F, Ford AC. Global prevalence of, and risk factors for, gastro-oesophageal reflux symptoms: a metaanalysis. Gut. 2018;67(3):430-440. https://doi.org/10.1136/gutjnl-2016-313589

4. Vakil N, van Zanten SV, Kahrilas P, Dent J, Jones R; Global Consensus Group. The Montreal definition and classification of gastroesophageal reflux disease: a global evidencebased consensus. Am J Gastroenterol. 2006;101(8):190020; quiz 1943. https://doi.org/10.1111/j.1572-0241.2006.00630.x

5. Yadlapati R, DeLay K. Proton Pump Inhibitor-Refractory Gastroesophageal Reflux Disease. Med Clin North Am. 2019;103(1):15-27. https://doi.org/10.1016/j.mcna.2018.08.002

6. Subramanian CR, Triadafilopoulos G. Refractory gastroesophageal reflux disease. Gastroenterol Rep (Oxf). 2015;3(1):41-53. https://doi.org/10.1093/gastro/gou061

7. Lin S, Li H, Fang X. Esophageal Motor Dysfunctions in Gastroesophageal Reflux Disease and Therapeutic Perspectives. J Neurogastroenterol Motil. 2019;25(4):499-507. https://doi.org/10.5056/jnm19081
8. Patel A, Posner S, Gyawali CP. Esophageal High-Resolution Manometry in Gastroesophageal Reflux Disease. JAMA. 2018;320(12):1279-1280. https://doi.org/10.1001/jama.2018.8694

9. Mello M, Gyawali CP. Esophageal manometry in gastroesophageal reflux disease. Gastroenterol Clin North Am. 2014;43(1):69-87. https://doi.org/10.1016/j.gtc.2013.11.005

10. Abdallah J, George N, Yamasaki T, Ganocy S, Fass R. Most Patients With Gastroesophageal Reflux Disease Who Failed Proton Pump Inhibitor Therapy Also Have Functional Esophageal Disorders. Clin Gastroenterol Hepatol. 2019;17(6):1073-1080.e1. https://doi.org/10.1016/j.cgh.2018.06.018

11. Stoikes N, Drapekin J, Kushnir V, Shaker A, Brunt LM, Gyawali CP. The value of multiple rapid swallows during preoperative esophageal manometry before laparoscopic antireflux surgery. Surg Endosc. 2012;26(12):3401-7. https://doi.org/10.1007/s00464-012-2350-0

12. Kahrilas PJ, Bredenoord AJ, Fox M, Gyawali CP, Roman $\mathrm{S}$, Smout AJ, et al. The Chicago Classification of esophageal motility disorders, v3.0. Neurogastroenterol Motil. 2015;27(2):160-74 https://doi.org/10.1111/nmo.12477

13. Katz PO, Gerson LB, Vela MF. Guidelines for the diagnosis and management of gastroesophageal reflux disease. Am J Gastroenterol. 2013 Mar; 108(3):308-28; quiz 329. https://doi.org/10.1038/ajg.2012.444

14. Gyawali CP, Kahrilas PJ, Savarino E, Zerbib F, Mion F, Smout AJPM, Vaezi M, Sifrim D, Fox MR, Vela MF, Tutuian R, Tack J, Bredenoord AJ, Pandolfino J, Roman S. Modern diagnosis of GERD: the Lyon Consensus. Gut. 2018;67(7):1351-1362. https://doi.org/10.1136/gutjnl-2017-314722 
15. Armstrong D, Marshall JK, Chiba N, Enns R, Fallone CA, Fass R, et al. Canadian Consensus Conference on the management of gastroesophageal reflux disease in adults - update 2004. Can J Gastroenterol. 2005;19(1):15-35. https://doi.org/10.1155/2005/836030

16. Patcharatrakul T, Gonlachanvit S. Gastroesophageal reflux symptoms in typical and atypical GERD: roles of gastroesophageal acid refluxes and esophageal motility. J Gastroenterol Hepatol. 2014;29(2):284-90. https://doi.org/10.1111/jgh.12347

17. Abdel Jalil AA, Castell DO. Ineffective Esophageal Motility (IEM): the Old-New Frontier in Esophagology. Curr Gastroenterol Rep. 2016;18(1):1. https://doi.org/10.1007/s11894-015-0472-y

18. Roman S, Kahrilas PJ. Management of spastic disorders of the esophagus. Gastroenterol Clin North Am. 2013;42(1):27-43. https://doi.org/10.1016/j.gtc.2012.11.002

19. Roman S, Pandolfino JE, Chen J, Boris L, Luger D, Kahrilas PJ. Phenotypes and clinical context of hypercontractility in high-resolution esophageal pressure topography (EPT). Am J Gastroenterol. 2012;107(1):37-45. https://doi.org/10.1038/ajg.2011.313

20. Roman S, Damon H, Pellissier PE, Mion F. Does body position modify the results of oesophageal high resolution manometry? Neurogastroenterol Motil. 2010;22(3):271-5. https://doi.org/10.1111/j.1365-2982.2009.01416.x

21. Roman S, Lin Z, Kwiatek MA, Pandolfino JE, Kahrilas PJ. Weak peristalsis in esophageal pressure topography: classification and association with Dysphagia. Am J Gastroenterol. 2011;106(2):349-56. https://doi.org/10.1038/ajg.2010.384

22. Bulsiewicz WJ, Kahrilas PJ, Kwiatek MA, Ghosh SK, Meek A, Pandolfino JE. Esophageal pressure topography criteria indicative of incomplete bolus clearance: a study using high-resolution impedance manometry. Am J

Gastroenterol. 2009;104(11):2721-8.

https://doi.org/10.1038/ajg.2009.467

23. Bogte A, Bredenoord AJ, Oors J, Siersema PD, Smout AJ. Relationship between esophageal contraction patterns and clearance of swallowed liquid and solid boluses in healthy controls and patients with dysphagia. Neurogastroenterol Motil. 2012;24(8):e364-72. https://doi.org/10.1111/j.1365-2982.2012.01949.x

24. Pu L, Chavalitdhamrong D, Summerlee RJ, Zhang Q. Effects of Posture and Swallow Volume on Esophageal Motility Morphology and Probability of Bolus Clearance: A Study Using High-Resolution Impedance Manometry. Gastroenterol Nurs. 2020;43(6):440-447. https://doi.org/10.1097/SGA.0000000000000356

25. Zizer E, Seufferlein T, Hänle MM. Impaired bolus clearance in combined high-resolution esophageal manometry and impedance measurement helps to differentiate between esophagogastric junction outflow obstruction and achalasia. Z Gastroenterol. 2017;55(2):129-135. English. https://doi.org/10.1055/s-0042-121267

26. Jain A, Baker JR, Chen JW. In ineffective esophageal motility, failed swallows are more functionally relevant than weak swallows. Neurogastroenterol Motil. 2018;30(6):e13297. https://doi.org/10.1111/nmo.13297

27. Zerbib F, Marin I, Cisternas D, Abrahao L Jr, Hani A, Leguizamo AM, et al. Ineffective esophageal motility and bolus clearance. A study with combined high-resolution manometry and impedance in asymptomatic controls and patients. Neurogastroenterol Motil. 2020;32(9):e13876. https://doi.org/10.1111/nmo.13876 